Review

\title{
Conformity without majority? The case for demarcating social from majority influences
}

\author{
Edwin J. C. van Leeuwen ${ }^{\text {a, b, c, * }}$, Daniel B. M. Haun ${ }^{\text {a, b, c }}$ \\ a Max Planck Institute for Psycholinguistics, Nijmegen, The Netherlands \\ ${ }^{\mathrm{b}}$ Max Planck Institute for Evolutionary Anthropology, Leipzig, Germany \\ ${ }^{c}$ Department of Developmental Psychology, University of Jena, Jena, Germany
}

\section{A R T I C L E I N F O}

\section{Article history:}

Received 9 May 2014

Initial acceptance 10 June 2014

Final acceptance 22 July 2014

Published online 16 September 2014

MS, number: $14-00383$

\section{Keywords:}

conformity

decision making

frequency-dependent learning

majority influence

nonhuman animals

social learning
In this review, we explore the extent to which the recent evidence for conformity in nonhuman animals may alternatively be explained by the animals' preference for social information regardless of the number of individuals demonstrating the respective behaviour. Conformity as a research topic originated in human psychology and has been described as the phenomenon in which individuals change their behaviour to match the behaviour displayed by the majority of group members. Recent studies have aimed to investigate the same process in nonhuman animals; however, most of the adopted designs have not been able to control for social influences independent of any majority influence and some studies have not even incorporated a majority in their designs. This begs the question to what extent the 'conformity interpretation' is preliminary and should be revisited in light of animals' general susceptibility to social influences. Similarly, demarcating social from majority influences sheds new light on the original findings in human psychology and motivates reinterpretation of the reported behavioural patterns in terms of social instead of majority influences. Conformity can have profound ramifications for individual fitness and group dynamics; identifying the exact source responsible for animals' behavioural adjustments is essential for understanding animals' learning biases and interpreting cross-species data in terms of evolutionary processes.

(C) 2014 The Association for the Study of Animal Behaviour. Published by Elsevier Ltd. All rights reserved.
Dating back to 1932, when Arthur Jenness observed that individuals' estimates of the number of beans in a jar were considerably influenced by the estimate of the majority of group members (Jenness, 1932), conformity has received extensive attention in human psychology. Defined by altering opinions or behaviour in order to match the majority, subsequent research robustly showed that a surprising number of the adult participants are conformists, even when the majority stance is conspicuously erroneous (Asch, 1956; Bond \& Smith, 1996; Sherif, 1936). In later years, it was shown that opting for the majority stance does not represent a biological conundrum, but may instead bestow substantial fitness benefits (Henrich \& Boyd, 1998; King \& Cowlishaw, 2007; Richerson \& Boyd, 2005; Wolf, Kurvers, Ward, Krause, \& Krause, 2013).

The tendency to adopt the behaviour of the majority of group members is considered to be one of the driving forces behind

\footnotetext{
* Correspondence: E. J. C. van Leeuwen, Department of Developmental Psychology, University of Jena, Am Steiger 3/1, 07743 Jena, Germany.

E-mail address: ejcvanleeuwen@gmail.com (E. J. C. van Leeuwen)
}

cultural diversification (Boyd \& Richerson, 1985; Henrich \& Boyd, 1998). By converging on the most prevalent conventions, groups move towards behavioural homogeneity while at the same time increasing the likelihood of emergent between-group heterogeneity: the hallmark of culture (Richerson \& Boyd, 2005). In recent years, majority influence has become a favourable research topic for behavioural biologists. One line of research has focused on our closest living relatives, the nonhuman primates (chimpanzees, Pan troglodytes: Bonnie, Horner, Whiten, \& de Waal, 2007; Haun, Rekers, \& Tomasello, 2012; Hopper, Schapiro, Lambeth, \& Brosnan, 2011; Whiten, Horner, \& de Waal, 2005; capuchin monkeys, Cebus apella: Dindo, Thierry, \& Whiten, 2008; Dindo, Whiten, \& de Waal, 2009; Perry, 2009), which could enable intriguing analysis of the evolutionary roots of this human phenomenon (MacLean et al., 2012). Another line of research has aimed to investigate the possibility of convergent evolution of conformity by focusing on phylogenetically more distant species, such as fish (Day, MacDonald, Brown, Laland, \& Reader, 2001; Kendal, Coolen, \& Laland, 2004; Pike \& Laland, 2010), rats (Galef \& Whiskin, 2008; Jolles, de Visser, \& van den Bos, 2011; Konopasky \& Telegdy, 1977) and fruit flies (Battesti, Moreno, Joly, \& Mery, 2012). 
However, as we aim to show in this review, most studies in both lines of investigation seemed to have overlooked the distinction between social and majority influences.

Specifically, in this review, we evaluate relevant conformity studies across animal taxa (including humans) to show that most designs have been insufficiently accurate to pinpoint whether subjects' behavioural adjustment was caused by social or majority influences. Important to note here is the logical distinction between social and majority influences: although majority influence is inevitably social influence, behavioural adjustment caused by social influences need not be caused by majority influence. For instance, individuals could discard their personal preference by copying alternative behaviour from just one other individual, as opposed to the majority of individuals. This distinction would need to be taken seriously if conformity, defined by not just social but majority influence, is to be studied validly. For this reason, first, we review recent conformity findings across nonhuman animals (henceforth 'animals') in light of the distinction between social and majority influences. Second, we revisit the essential details of the human psychology studies that gave rise to the conformity definition and explore whether their findings could be explained in terms other than majority influence. Lastly, we conclude with suggestions to translate the definitional distinction between social and majority influences into empirical study designs. To be able to interpret the intriguing social phenomenon of 'conformity' in evolutionary terms, or identify the exact learning mechanisms present in species' behavioural repertoires, we need to calibrate the conformity designs across research disciplines.

To avoid confusion, it is important to explicitly distinguish between the conformity definitions that emerged from the study of human psychology and cultural evolution (see Morgan \& Laland, 2012). The human psychology definition emphasizes the fact that individuals have to forgo their behavioural inclination, thereby adopting the behaviour of the majority (e.g. Asch, 1956; Jenness, 1932; Sherif, 1936). Thus, in this case, individuals possess a certain behaviour or conviction before being exposed to the conflicting stance of the majority. The cultural evolutionary approach, by contrast, investigates cost/benefit scenarios in which it would be beneficial (in terms of fitness consequences) for individuals to learn socially instead of individually, and has shown that in many cases where individuals are predicted to learn socially, they would favour not just any social information, but the information specifically transmitted by the majority of interactants (Boyd \& Richerson, 1985; Henrich \& Boyd, 1998; also see Aoki \& Feldman, 2013). In other words, the cultural evolutionary approach analyses individuals on the verge of obtaining their first behavioural strategy, not individuals discarding their familiar behaviour. Thus, where the psychological approach to conformity focuses on the determinants of knowledgeable individuals' tendency to adjust their behaviour to the majority, the cultural evolutionary approach typically models the likelihood of naïve individuals obtaining the most common cultural variant. This subtle distinction could have important ramifications for understanding behavioural patterns indicative of conformity: where individuals in possession of a functional and/or preferred behavioural strategy might be more inclined to persevere in using their strategy than adopting random social information, individuals that are already predicted to learn socially could end up with the majority behaviour through exactly this process of random copying (e.g. see Mesoudi, 2009). We elaborate on this distinction in the section on human conformity findings. Furthermore, while the human psychology approach has traditionally been more concerned with immediately influential variables such as group size and task ambiguity (e.g. see Bond, 2005), the cultural evolutionary approach has mainly been concerned with the broader ramifications of behavioural decisions for the evolution of culture, expressed as relative within-group homogeneity and betweengroup heterogeneity (e.g. see Richerson \& Boyd, 2005). For the reason that we are interested in the extent to which conformity can be identified through specific experimental features (i.e. the manipulation of group size) and dissected in social and majority influences, in this review we focus entirely on the human psychology operationalization of conformity.

\section{DO PRIMATES SHOW CONFORMITY?}

Most studies on conformity in animals have been conducted in nonhuman primates (henceforth 'primates'). On the one hand, the label 'conformity' has been used to describe the process by which primates obtain the strategy that gradually becomes the majority strategy. Starting with one skilled individual (natural invention or trained skill), the rest of the group acquires the same particular way of behaving by means of social learning (Hopper et al., 2011; Perry, 2009; Whiten et al., 2005). On the other hand, the label 'conformity' has been used to describe the process by which primates would revert back to the majority strategy after discovering an alternative strategy (chimpanzees: Bonnie et al., 2007; Hopper et al., 2011; Whiten et al., 2005; capuchin monkeys: Dindo et al., 2008, 2009). Here, after socially acquiring a particular way of behaving, some individuals would occasionally explore an alternative strategy, but then change back to preferring the majority strategy.

The interpretation of this latter behavioural pattern in terms of conformity has been criticized based on the fact that the same behavioural pattern could be explained by conservatism (i.e. the reluctance to switch techniques once one technique has been proficiently mastered; Hrubesch, Preuschoft, \& van Schaik, 2009; van Leeuwen, Cronin, Schütte, Call, \& Haun, 2013; van Leeuwen \& Haun, 2013; Pesendorfer et al., 2009). In that case, the reconvergence to the majority behaviour might not even be socially mediated (see van Leeuwen \& Haun, 2013). More relevant to the current focus, however, are the following issues.

First, in the case where conformity was claimed based on gradual behavioural convergence (e.g. Hopper et al., 2011, p. 6), for at least half of the group members there could not have been a majority present to conform to. In other words, for the first half of the group members, this gradual diffusion of information over individuals cannot possibly be attributed to conformity as it is typically defined. The second half of naïve learners could technically have been influenced by the majority (if all individuals of the first half adopted the behaviour demonstrated by the initiator); however, the social-learning process that was sufficient for the first half of the group to learn the target behaviour cannot be filtered out as an explanatory variable. More importantly, in this stage, none of the subjects have adjusted their behaviour to the majority; they have 'merely' acquired the behaviour that gradually becomes the majority strategy. This means that in this diffusion process, the two main features of the conformity phenomenon are compromised: copying the majority and forgoing individually acquired behaviour. In this light, the pattern in which subjects revert back to the majority strategy after discovering an alternative may have more grounds to claim conformity (i.e. in this case it could be argued that an established behaviour needs to be discarded). However, in these reversion studies, where subjects reconverged on the majority strategy after exploring an alternative strategy, it remained unexplored whether the reverting individuals had had the chance to actually observe the majority and questionable to what extent reverting back to the first-learned strategy entails forgoing individually acquired behaviour (see van Leeuwen \& Haun, 2013). Moreover, a recent study found that chimpanzees who perceived a majority of group members engaging in a different (yet equally effortful and rewarding) strategy from themselves did not adjust 
their behaviour to the majority (van Leeuwen et al., 2013). Contrary to the earlier mentioned conformity studies in chimpanzees (e.g. Hopper et al., 2011; Whiten et al., 2005), van Leeuwen et al. (2013) did not equate the majority strategy with the first-learned strategy of the minority chimpanzees, which enabled them to conclude that chimpanzees prefer their familiar strategy over conforming to the majority. Another recent study, however, seems to demonstrate nicely that primates can both be adopting the majority strategy and forgoing their previously established behavioural preference, the two pillars of the conformity definition (van de Waal, Borgeaud, \& Whiten, 2013). In this study on wild vervet monkeys, Chlorocebus aethiops, both maturing and immigrating individuals adopted the foraging preference shared by the majority of the local group, indicating that at least the immigrants show conformity as it is defined in human psychology (van de Waal et al., 2013). However, the claim for selective majority copying remained circumstantial at most (authors' own conclusion, see supplementary materials in van de Waal et al., 2013). Indeed, the fact that most of the resident vervet monkeys used the foraging strategy to which the immigrants 'conformed' does not mean that the immigrants used this majority information in their foraging decisions. Recognizing that learning entails a complex arena of individual, social and contextual characteristics (e.g. see Kendal, Coolen, \& Laland, 2009), it might be that other learning biases have been at play during the acclimatization process of the immigrating males, such as a bias to value social information over individual information when the environment is uncertain (where a majority bias need not be at play).

The second issue that pertains directly to our focus of demarcating social from majority influences is related to the finding that chimpanzees who have acquired their behaviour socially have been shown to be relatively rigid in the use thereof, even at the cost of efficiency (Price, Lambeth, Schapiro, \& Whiten, 2009). Importantly, Price et al. (2009) obtained these results in the scenario where the chimpanzees learned from one conspecific, not from a majority. A similar finding was reported by Whiten (1998): in this study, chimpanzees preferentially used their first-learned action pattern that had been demonstrated to them by one human experimenter, even after discovering that other sequences worked equally well (Whiten, 1998). These observations suggest that chimpanzees remain faithful to what they have learned first, or socially, and that these characteristics, not majority influences, can sufficiently account for the information diffusion and the reversion pattern that form the foundation of the conformity interpretation in primates (also see Hrubesch et al., 2009; Pesendorfer et al., 2009; van Leeuwen \& Haun, 2013).

\section{DO RATS SHOW CONFORMITY?}

The conformity data in rats, Rattus norvegicus, are also a good example of the potent effects that social information can exert without it being transmitted by a majority of individuals. In an early conformity study, Konopasky and Telegdy (1977) showed that some rats would follow the unrewarded strategy option demonstrated to them by one 'leader' rat, even when they were experienced in choosing the rewarded option. More recent studies used a similar design in which 'observer' rats were shown to copy the foraging decision of one demonstrator rat, even when they had experienced those options to be unpalatable or even toxic (Galef \& Whiskin, 2008; Jolles et al., 2011). In an attempt to shed light on the distinction between social and majority influences, Galef (2009) pointed to the findings in which naïve rats showed an increased probability of adopting a demonstrated pattern of behaviour as the proportion of demonstrators increased (e.g. Chou \& Richerson, 1992; Galef \& Whiskin, 1995; see Galef, 2009). However, paraphrasing Galef's (2009) own conclusion, in the case of naïve individuals, the bias towards copying the majority can only be identified when the probability of adopting the majority strategy increases disproportionately compared with the increase in the relative majority size, which has not shown to be the case in any of the rat studies (Galef, 2009; Laland, 2004). In other words, the naïve rats may have responded to the amount of social information, regardless of it being transmitted by a majority of group members. More importantly for this review, however, is the fact that none of these latter studies investigated the tendency of knowledgeable rats to adopt social information, which invalidates an adequate comparison to the findings of the original human conformity studies.

\section{DO FISH SHOW CONFORMITY?}

Studies on conformity in fish have followed different approaches from experiments in other taxa and thus allow for slightly different interpretations. An early study by Day et al. (2001) showed that guppies, Poecilia reticulata, are hindered in their ability to locate food resources by the relative inertia of larger groups when visibility is compromised. The authors concluded that the guppies increasingly preferred to remain part of the group with increasing group size, and hence interpreted their findings in terms of 'positive frequency-dependent social learning, or conformity' (Day et al., 2001, p. 924; also see Lachlan, Crooks, \& Laland, 1998; Laland \& Williams, 1997 for similar findings and interpretations). This interpretation, however, has been criticized based on the consideration that guppies are a shoaling species and therefore let their behavioural choices become immersed in the collective group decision. In other words, as individual guppies by default prefer to swim in groups, their choices in such a group scenario, even if they were affected by majority influences, would be overshadowed by their shoaling preference (see Claidière \& Whiten, 2012). Whether this critique is accepted or not, notwithstanding the interesting social effects revealed in this study, the fact that the guppies were not tested in a situation in which they needed to forgo their personal knowledge compromises a valid comparison between the guppies' behaviour and the concept of conformity as originally defined in human psychology.

In an attempt to investigate this latter operationalization (conformity sensu Asch, 1956), researchers tested whether guppies would forgo their individually acquired information on food patch profitability under the influence of majority demonstrations. Although naïve subjects followed the majority convincingly, the knowledgeable subjects did not opt for exploring the food patch demonstrated by the majority, unless their individually acquired strategy was made relatively costly (Kendal et al., 2004). These findings indicate that guppies are susceptible to social information. However, owing to the conspicuous inequality in net strategy payoff (i.e. only the individually acquired strategy was made costly), they do not yet shed light on guppies' inclination to conform. In fact, the guppies could have chosen the majority option because it was energetically more efficient than their individually acquired strategy. Moreover, it remains to be investigated to what extent the guppies would have opted for the demonstrated food patch if there had been only one demonstrator (as in the rat studies).

More convincing evidence for conformity in fish comes from a study in sticklebacks. Pike and Laland (2010) showed that ninespined sticklebacks, Pungitius pungitius, adjusted their food patch preference to the conflicting preference of the majority of demonstrators, contingent on the increasing (relative) majority size (Pike \& Laland, 2010). In contrast to the studies in guppies, this stickleback study shows that fish may not just favour social information over individually acquired information, but may be 
specifically drawn to synchronizing their behaviour with the largest group of dissidents.

\section{DO BIRDS SHOW CONFORMITY?}

To our knowledge, claims of conformity in birds have not been made explicitly. However, several bird species have been shown to adjust their behaviour upon observing conspecifics demonstrating alternative preferences in a similar fashion to the rats in the aforementioned studies. For instance, indigo buntings, Passerina cyanea, were found to adjust features of their songs to the songs of nearby individuals (Payne, 1985) and zebra finches, Taeniopygia guttata, adopted the foraging and mating preferences of conspecifics, even when this behaviour conflicted with their personal preferences (Rosa, Nguyen, \& Dubois, 2012). Other studies implicitly corroborate these findings, for instance by showing that birds are likely to adopt local song dialects (e.g. Espmark, Lampe, \& Bjerke, 1989; Mundinger, 1982) and breeding site preferences (e.g. Boulinier, McCoy, Yoccoz, Gasparini, \& Tveraa, 2008; Brown, Bomberger Brown, \& Danchin, 2000; Danchin, Boulinier, \& Massot, 1998). However, although this kind of behavioural adjustment can lead to local behavioural convergence (i.e. a possible signature of conformity), the actual bias towards copying not just any demonstrated behaviour but the behaviour performed by the majority of group members remains to be demonstrated (e.g. see Haun, van Leeuwen, \& Edelson, 2013).

\section{DO INSECTS SHOW CONFORMITY?}

The most relevant data from the insect literature for our discussion revolving around conformity comes from studies in which personal information was pitted against social information. For instance, it was recently shown that female fruit flies, Drosophila melanogaster, preferred to mate with poor-condition rather than good-condition males after observing the poor-condition but not good-condition males in close proximity with another female (i.e. indicating that those males were previously chosen by other females; Mery et al., 2009). Female fruit flies in control conditions (without any social information) showed a preference for goodcondition males. In conjunction, these findings indicate that female fruit flies adjusted their mating behaviour to social information against personal preference. Another recent study showed that female fruit flies modified their oviposition preference after observing eight female conspecifics unanimously expressing interest in one particular (arbitrary) egg-laying medium (Battesti et al., 2012). Control conditions were used to establish that interaction with the demonstrators was necessary (and sufficient) to induce behavioural acquiescence in the observer flies, and as prior personal sampling had endowed the observers with the knowledge that both media were equally rewarding, these findings were interpreted as adjusting mating behaviour against personal preference.

Notwithstanding the value of these studies for our understanding of animals' individual and social information use, these results confirm the central argument of this review: social information can induce behavioural adjustment irrespective of majority influences. The fruit fly studies addressed above have impressively shown that social information can be a potent vector in the decision-making process of animals, even for those with 'miniature brains' (Leadbeater \& Chittka, 2007b), but do not necessarily tie this social influence to a majority bias; whereas the first study only used single demonstrators (Mery et al., 2009), the latter did not quantify the impact of single or minority demonstrators, which would seem a necessary benchmark for interpreting the observed social influences in terms of majority influence (Battesti et al., 2012).
The (social) insect literature alludes to at least two more intriguing avenues. First, the fruit fly studies point to the importance of operationalizing the 'distance' between subjects' original preference and the demonstrated alternative. For instance, the difference between poor- and good-condition males (see Mery et al., 2009) could plausibly be considered of a different order than the difference between a recently learned arbitrariness for egg-laying media and the demonstration of the usage of one particular medium by conspecifics (see Battesti et al., 2012). In the following sections, we propose that information on this distance measure, the extent to which subjects' pre-established behaviour deviates from the socially observed behaviour, is necessary for interpreting the magnitude of behavioural adjustments, including conformity. Second, researchers studying information use in bee species (e.g. Apis mellifera and Bombus terrestris) consistently approach decision making as an intricate process involving many related aspects, such as the cost of individual sampling, the success of individual information-gathering attempts and whether social information yields new or familiar knowledge (Biesmeijer \& Seeley, 2005; Grüter, Leadbeater, \& Ratnieks, 2010; Leadbeater \& Chittka, 2005, 2007a). This approach is more in line with social influence theories (Latane \& Wolf, 1981; Tanford \& Penrod, 1984) than with the paradigmatic conformity interpretations where social influence is coarsely collapsed into 'the majority' (see Asch, 1956; Sherif, 1936). In the next sections, we address these two decisionmaking approaches in more detail (i.e. conformity sensu Asch, 1956 and the social influence perspective sensu Latane \& Wolf, 1981 and Tanford \& Penrod, 1984).

\section{HUMAN CONFORMITY REVISITED}

Although the conformity phenomenon as a research topic stems from the realm of human social psychology, the evidence in favour of human conformity remains ambiguous. One aspect that may have contributed to this ambiguity concerns the details of the social-information source that induces conformity, where doubt has been cast on the claim that the feature 'majority' is pivotal to conformity (see Bond, 2005). Here, instead of presenting a general overview of the conformity findings in humans (reviewed in e.g. Bond, 2005; Haun et al., 2013; Morgan \& Laland, 2012) and in order to facilitate valid cross-species comparisons, we scrutinize the paradigmatic human conformity studies in the same light of social and majority influences as the animal studies reviewed before.

In the seminal human psychology experiments conducted by Solomon Asch, in which subjects were asked to indicate which line (out of three) matched a given target-line in length, a consistent proportion of the adult subjects adjusted their individual opinion to the erroneous conviction of (at least) three confederates (see Asch, 1951, 1955, 1956). Asch consistently referred to this behavioural phenomenon in terms of majority influences (e.g. Asch, 1956), which as such was adopted by contemporary scientists (e.g. Allen, 1965; Cohen, Bornstein, \& Sherman, 1973) and has remained a commonly used concept (e.g. see Bond, 2005). In line with the tenet of the current review, revisiting this behavioural phenomenon in light of the demarcation between social and majority influences brings up to two important aspects. First, it needs to be emphasized that in the Asch studies (Asch, 1951, 1955, 1956) the 'distance' between the subject's original stance and the demonstrated alternative seems relatively large compared with most of the nonhuman conformity studies addressed here. Although this may seem trivial, it is our contention that the magnitude of conformity can only be adequately assessed when this distance is known or at least somehow operationalized (Starkweather, 1970; also see van Leeuwen \& Haun, 2013). For instance, for each subject in the Asch studies, the distance between his/her initial line judgement and the 
conflicting judgement of Asch's confederates (e.g. see Asch, 1956) might arguably be larger than the distance between the two equally effortful and productive token reward alternatives for each chimpanzee in the most cited chimpanzee conformity study (Whiten et al., 2005). Whereas the former distance exists between easily perceivable, factual stances (i.e. one line is objectively 'correct'), the latter distance is arbitrary with both options (individual versus majority) being equally likely to be 'correct' (i.e. there is no truth value inherent to the alternatives). This means that in these specific designs, it might take more social/majority influence to make the human subjects conform than it would take to make the chimpanzees conform. As such, this distance measure would be a valuable addition to the study of conformity (also see section 'Conclusion and future directions'). Moreover, identifying the inherent presence of this distance feature is useful for illustrating the meaning of the second aspect, which might be best described by the following question: 'do the subjects in the seminal social psychology studies conform because the alternative is demonstrated by the majority or do they give in to social influences (where again it should be noted that majority influences are inevitably social influences, whereas social influences do not have to be majority influences)?'. To answer this question, it may be essential to adopt the logical view that social information, just like individually acquired information, can have an intrinsic value apart from the value that subjects may attribute to 'the majority'. In other words, social information does not become informative and/or meaningful only when it is presented by a majority of group members; it has an impact on social animals regardless of this majority feature. Proponents of social-influence theories have adopted and found empirical support for this argument in humans (e.g. see Latane \& Wolf, 1981; Tanford \& Penrod, 1984). They have additionally posed that not the mere fact that individuals are confronted with a majority, but more nuanced parameters such as strength and immediacy of the social-influence sources and the number of demonstrators have an impact on the behavioural decision of the subjects. Similarly, animal studies have shown that species differ in their reliance on social information (Coolen, van Bergen, Day, \& Laland, 2003; van Leeuwen, Call, \& Haun, n.d.) and that also other factors such as social-rearing environment and experienced stress can affect animals' tendency to use social cues (guppies: Chapman, Ward, \& Krause, 2008; Japanese quail, Coturnix japonica: Boogert, Zimmer, \& Spencer, 2013). Taken together, these findings indicate that social influence is a vector of which the magnitude is determined by many more features than merely 'the majority'. In turn, this underlines the central claim of this review: differentiating between the effects of social and majority influences is necessary for validly interpreting 'conformity' findings and thus for providing a calibrated platform to compare conformity across species. For instance, the aforementioned studies in rats seem to indicate that subjects could adjust their previously learned (and even preferred) behaviour based on the information demonstrated by just one conspecific (Galef \& Whiskin, 2008; Jolles et al., 2011). This finding shows that in a given species, social information can outweigh individual information irrespective of any majority bias. In turn, this finding implies the more general premise that in any species the values of social and individual information exist within an intricate trade-off that can, but does not necessarily have to, be moderated by the feature 'majority' (e.g. see Kendal et al., 2009; Kendal, Coolen, van Bergen, \& Laland, 2005). In other words, if the value attributed to social information trumps the value attributed to individual information, then behaviour and/or convictions could be adjusted through the pull of social information without any majority influence. For instance, in the absence of any susceptibility to majority influences, animals could have evolved or learned preferences for social over individual information, or adjust their behaviour when social information provides a more recent sample of the environment than individual information.

Note that the distance feature and the possibility of having social but not majority influences at play are nicely reflected in the results from the Asch studies. Recapitulating, in these studies, subjects were asked to indicate which line (out of three) matched a given target-line in length (e.g. see Asch, 1956). The discrepancy between the subject's judgement and the intentionally erroneous judgement conveyed by Asch's confederate(s) might be considered large here because the task at stake is about an easily perceivable and straightforward factual quandary, i.e. subjects can be relatively sure of the correct answer. As such, the subjects may need more social weight in order to be swayed than one confederate could provide. If this premise is accepted, additional social weight would be needed to make subjects adjust their opinion to the conflicting stance, which is consistent with the finding that at least three confederates unanimously need to express this conflicting position (e.g. Asch, 1956). Besides an illustration of how the distance measure could be tied to conformity, this also indicates that the increased social pressure could have been responsible for subjects' behavioural adjustment, not 'the majority' per se. Similarly, if the distance measure is meaningful, subjects should be more amenable to conform to the confederate(s) when the two conflicting stances differ less in their accuracy/profitability. This seems to be supported by the recent finding that 2-year-old children conform equally to one or three demonstrators when the two 'conflicting' strategies are equal on every level except for arbitrary location (i.e. which identically shaped pipe to drop a token into in order to receive a reward; Haun, Rekers, \& Tomasello, in press).

The distinction between social and majority influences is further illustrated by some details of the original Asch studies: Whenever the focal subject finds himself not being the only dissenter from the majority conviction, conformity breaks down drastically, even when the other dissenter does not agree with the focal subject but instead indicates the third alternative judgement (Asch, 1956). Similar observations were made when the majority comprised only two confederates: the extent to which focal subjects conformed to the majority declined drastically in such scenarios (see Asch, 1951, $1955,1956)$. Note that in both cases, the majority remained present, but was not conformed to. Minimally, these observations indicate that other features of social influences might have been at play and that the moderator of conformity 'to a majority' might be poorly grounded (see Mesoudi, 2009 for a more detailed analysis of the difficulty to attribute subjects' 'conformity' in the Asch studies to the majority).

Alluded to in previous sections, one stringent attempt has been made to pinpoint selective majority copying in the context of conformity research. This attempt stems from the cultural evolutionary framework, where the seminal work of Boyd and Richerson showed that in order to identify the bias to conform to the cultural variant that is used by the majority, one needs to show that the likelihood of copying the majority behaviour for a given individual increases disproportionately compared with the increase of the relative majority size (Boyd \& Richerson, 1985; Richerson \& Boyd, 2005). For instance, when the relative majority size increases from 0.6 to 0.8 (proportion of the population), the likelihood with which a majority biased individual copies the majority would increase significantly more than $33.3 \%$. The reasoning behind this criterion is that individuals who would copy the majority increasingly, but not to a disproportionate extent, could similarly copy any individual in the population randomly, thus without any selective majority considerations (see Henrich \& Boyd, 1998; van Leeuwen \& Haun, 2013; Mesoudi, 2009). One recent study on human adults' decision-making processes integrated this mathematical criterion into their analyses and found a substantial part of the subjects to be 
majority biased (Morgan, Rendell, Ehn, Hoppitt, \& Laland, 2012). However, while this study impressively shows that humans rely on increasingly large majorities in the face of uncertain contingencies (which strategy leads to the highest rewards?), it only minimally tapped into subjects' inclination to forgo their prior behaviour as strategies were instantly chosen and the most rewarding strategy remained unknown until after the decision was made. This means that the individuals concerned did not have pre-established behavioural patterns that needed to be discarded; they rather assessed and reassessed their strategy upon each decision-making trial (in a way, this would be equal to a distance estimate being close to zero). In the current review we aim to clarify that the aspect of abandoning previously ingrained or preferred behaviour makes for a different information acquisition context from the cultural evolutionary definition of conformity in which naïve individuals are yet to establish their first behavioural pattern (i.e. when mathematically predicted to learn socially instead of individually, will majority considerations guide individuals' copying preferences?). One intuitive consideration that might clarify this difference pertains to the copying likelihoods: whereas the naïve individuals in the cultural evolutionary definition are already expected to copy behaviour from their group members, the very question of whether an individual adopts the presented social information forms the core of the human psychology definition. This means that where the naïve individuals already have a likelihood higher than 0.5 of ending up with the cultural variant of the majority based on 'random copying' (the majority by definition comprises more than half of the individuals of the population), the knowledgeable individuals might be expected to stay true to their prior knowledge, especially in cases where the presented social information is conspicuously erroneous (as in the Asch studies, e.g. Asch, 1956). Thus, adopting social information might reflect different underlying decision-making processes in these two scenarios (with the earlier discussed distance measure as a moderating variable for the magnitude of the decision-making process in the human psychology, but not the cultural evolutionary context of conformity). A related difference between the cultural evolutionary and human psychology approach to conformity pertains to their level of analysis: Where the former operationalization focuses on transmission biases and their population-level ramifications (e.g. conformist transmission leading to within-group homogeneity and between-group heterogeneity: Boyd \& Richerson, 1985; Henrich \& Boyd, 1998), the latter focuses on processes mediating behavioural responses on the individual level, which requires more specific psychological scrutiny (e.g. What is the value attributed to individual knowledge? What is the perceived efficacy of the socially demonstrated option?; see Kendal et al., 2009; Perreault, Moya, \& Boyd, 2012; Rieucau \& Giraldeau, 2011).

In sum, within the paradigmatic conformity operationalization in the study of human psychology, we have outlined similar difficulties in identifying the exact social source that is conformed to as in most animal studies. In the final section, we draw conclusions based on our review and delineate conceptual and empirical ways to demarcate social from majority influences and thus clarify the study of conformity.

\section{CONCLUSION AND FUTURE DIRECTIONS}

In this review, we have advocated the necessity of demarcating social influences from majority influences by showing that most of the animal conformity studies have confounded these related vectors. Whereas some conformity studies have not even incorporated a majority in their designs, others have not been able to control for social influences independent of the majority influence (cf. Pike \& Laland, 2010). Even in the seminal human psychology studies (e.g. Asch, 1956), it remains unclear whether the subjects conformed under mere social or actual majority influences. This begs the question of to what extent the 'conformity interpretation' in most of the studies reviewed here is justified and should be revisited in light of animals' general susceptibility to social influences. The most important reason for doing so is empirical accuracy: only by identifying which social aspect feeds into the perception and subsequent behaviour of animals will we be able to discover which learning biases are pivotal in a species' repertoire and compare these behavioural tendencies across species.

A theoretical and empirical distinction between social and majority influences is valuable, as it may prevent scholars from unwittingly interpreting social influences in terms of conformity (e.g. see van den Bos, Jolles, \& Homberg, 2013). When scholars adhere to the cultural evolutionary definition of conformity, results should show that animals are disproportionately inclined to copy increasingly large relative majority sizes, while the aspect of 'forgoing preestablished behaviour' can accordingly be disregarded (but see Strimling, Enquist, \& Eriksson, 2009 for an important first approach to incorporating individuals' reluctance to switch strategies into models of cultural evolution). However, when the human psychology definition of conformity is used, this very aspect of forgoing preestablished behaviour is central to the analysis and scholars should provide evidence that this pre-established behaviour is the subjects' default strategy in the presence of alternatives but in the absence of conspecifics (thus forming one side of the distance measure; see previous section). To keep these two definitions apart, in earlier work, we have explicitly labelled the former definition 'conformist transmission' and the latter definition 'conformity', thereby using the labels coined in the respective research fields themselves (cultural evolution: e.g. Boyd \& Richerson, 1985; Henrich \& Boyd, 1998; human psychology: e.g. Asch, 1956; see Haun et al., 2013). Importantly, in both approaches to 'conformity', the distinction between social and majority influence is necessary for empirical accuracy. In the cultural evolutionary case, social influences are typically equated with 'random copying', which means that conspecifics are being imitated (i.e. evidence of social influence), but through an unbiased instead of a majority-biased strategy. This unbiased strategy yields behavioural convergence proportionate to the size of the majority (see previous section), but fails to generate structural between-group variation: the very reason why scholars studying cultural evolution are interested in conformist transmission (e.g. see Boyd \& Richerson, 1985). Thus, here, behavioural effects caused by social influences preclude interpretation in terms of 'conformist transmission' (e.g. see Laland, 2004; Richerson \& Boyd, 2005). In the human psychology approach, forming the framework of this review, social influences may cause individuals to forgo their preestablished behaviour even when there is no majority present to conform to, which is clearly exemplified in the rat studies (Galef \& Whiskin, 2008; Jolles et al., 2011). As such, the behavioural adjustments observed in rats after being exposed to one conspecific would not be considered conformity in the sense of the original operationalization (i.e. where the adjustment needs to be caused by the majority). In fact, this review has aimed to show that these social influences may have been underlying the purported conformity effects in many studies, even in the seminal human psychology ones.

The assumption that social instead of majority influences have affected observers, in some cases, seems to be straightforwardly confirmed by the fact that the conformity effect was already observed when the target individual was confronted with only one conflicting example (e.g. Galef \& Whiskin, 2008; Haun et al., in press; Jolles et al., 2011). Other cases remain inconclusive, as only a majority scenario was tested (e.g. Battesti et al., 2012; van de Waal et al., 2013; Whiten et al., 2005). What would have been observed if the subjects in these studies had been additionally tested in a 
single-model scenario? And if the subjects had adjusted their behaviour already in this single-model scenario, similar to the rats, what would have been the conclusion? In yet other cases, it became evident that the scope of social influences can stretch from the effect of one individual to the effect of many individuals before it turning into a majority influence (see previous section). For instance, three individuals may exert more social influence than one individual, but they still do not have to be the majority. In fact, it may be quintessential to acknowledge that these three individuals exert substantial social influence even when they are the majority (e.g. see Boyd \& Richerson, 1985; Mesoudi, 2009). Furthermore, social influence can be more or less pressing depending on modifiers such as species, salience or perceived efficacy (e.g. Latane \& Wolf, 1981; Tanford \& Penrod, 1984; see also van Bergen, Coolen, \& Laland, 2004; Boogert et al., 2013; Chapman et al., 2008; Coolen et al., 2003; Kendal et al., 2009). Thus, social influence is not a fixed effect, but a contingent vector with an associated magnitude. In more detail, the impact of social influence in any given situation is dependent on a plethora of ultimate and proximate determinants (e.g. species, life history, the characteristics of the model, previous social-learning experiences and the associated stakes of acquiring information; see also Laland, 2004; Kendal et al., 2009). To evidence conformity as the behaviouraladjustment effect caused by the majority, the effects of social influences need to be filtered out.

Importantly, all the studies addressed in this review have provided valuable insights into the extent to which species incorporate socially demonstrated behaviour into their repertoires. The only proposition that we would like to advance based on the considerations related to social and majority influences, however, entails a more rigid specification of the social-learning features at play in any learning trade-off study. Especially in the case of conformity, we envision that definitional and empirical ambiguity could be reduced by elaborating on the exact source of behavioural adjustment. In principle, this could be done by describing the design details in relation to the specific social-learning bias under study. For instance, when studying conformity, efforts should be made to specify how the employed design differentiates between the majority bias, social influence (with its species- and contextdependent estimate) and biases pertaining to the characteristics of the individuals in the majority (e.g. kin, skill, prestige/dominance). Empirically, it could be a helpful heuristic to always design a conformity study such that the effects of both the majority and a single demonstrator are assessed. The results of such a comparison would already coarsely indicate whether a 'group consideration', a central feature of the conformity definition, is necessary to explain the results. In other words, if animals would already adjust their behaviour to match a single demonstrator, then clearly their acquiescence to a group of demonstrators (e.g. to a majority) could not be unequivocally interpreted in terms of conformity.

Additionally, we propose acknowledging the 'distance' between the pre-established behaviour of the focal subject and the behavioural alternative presented by the majority as an important conformity variable. For instance, only by knowing parameters of the original stance of the focal subject (e.g. in terms of reinforcement history or relative preference) will its conformity become interpretable. One way to approach this aspect objectively might be to quantify the extent to which the focal subject persists in its stance in the presence of the alternatives, but in the absence of conspecifics. In the setting of the earlier mentioned line judgement task, during such a quantification, subjects will probably persist with high fidelity in choosing the correct line (see Corriveau \& Harris, 2010). On the other hand, when confronted with two equally effortful and rewarding alternatives (e.g. the chimpanzees in Whiten et al., 2005), subjects may show a less pronounced (pre- established) preference for a given strategy. While such a settingspecific quantification may be an elaborate undertaking, it seems highly plausible that subjects' responses to social demonstrations are mediated by the magnitude of their initial preference.

Finally, we acknowledge that different motivations to conform have been identified. In their influential work, Deutsch and Gerard (1955) proposed using 'informational conformity' for cases where people conform because of their conviction that others comprise a trustworthy source of information about reality, and 'normative conformity' for cases where people conform in anticipation of inducing positive feelings in others (Deutsch \& Gerard, 1955, also see Claidière \& Whiten, 2012; van Schaik, 2012). As we aimed to focus specifically on clarifying the study of the social source to which subjects conform, we have not treated this motivational dimension in this review. However, this distinction between motivations is compatible with our proposed scrutiny. For instance, it could be found that subjects adjust their behaviour to 'one conspecific' for 'informational' reasons, or that subjects conform to 'a group of three strangers' for 'normative' reasons. Any combination of social source and motivation could in principle be possible, yet where the study of conformity motivations has been constructively delineated (see Claidière \& Whiten, 2012), with this review, we hope to have similarly advanced the need and structure for adequately identifying the social source responsible for individuals' behavioural adjustments.

\section{Acknowledgments}

This work was supported by the Comparative Cognitive Anthropology research group of the Max Planck Institute for Psycholinguistics. We are grateful to Katherine Cronin, Alex Mesoudi and three anonymous referees for helpful comments on the manuscript.

\section{References}

Allen, V. L. (1965). Situational factors in conformity. Advances in Experimental Social Psychology, 2, 133-175.

Aoki, K., \& Feldman, M. W. (2013). Evolution of learning strategies in temporally and spatially variable environments: a review of theory. Theoretical Population Biology, 90, 64-81.

Asch, S. E. (1951). Effects of group pressure upon the modification and distortion of judgment. In H. Guetzkow (Ed.), Groups, leadership and men (pp. 117-190). Pittsburgh, PA: Carnegie Press.

Asch, S. E. (1955). Opinions and social pressure. Scientific American, 193, 31-35.

Asch, S. E. (1956). Studies of independence and conformity. 1. A minority of one against a unanimous majority. Psychological Monographs, 70, 1-70.

Battesti, M., Moreno, C., Joly, D., \& Mery, F. (2012). Spread of social information and dynamics of social transmission within Drosophila groups. Current Biology, 22, 309-313.

van Bergen, Y., Coolen, I., \& Laland, K. (2004). Nine-spined sticklebacks exploit the most reliable source when public and private information conflict. Proceedings of the Royal Society B: Biological Sciences, 271, 957-962.

Biesmeijer, J. C., \& Seeley, T. D. (2005). The use of waggle dance information by honey bees throughout their foraging careers. Behavioral Ecology and Sociobiology, 59, 133-142.

Bond, R. (2005). Group size and conformity. Group Processes \& Intergroup Relations, $8,331-354$

Bond, R., \& Smith, P. B. (1996). Culture and conformity: a meta-analysis of studies using Asch's (1952b, 1956) line judgment task. Psychological Bulletin, 119, $111-137$.

Bonnie, K. E., Horner, V., Whiten, A., \& de Waal, F. B. M. (2007). Spread of arbitrary conventions among chimpanzees: a controlled experiment. Proceedings of the Royal Society B: Biological Sciences, 274, 367-372.

Boogert, N. J., Zimmer, C., \& Spencer, K. A. (2013). Pre- and post-natal stress have opposing effects on social information use. Biology Letters, 9. http://dx.doi.org/ 10.1098/rsbl.2012.1088.

van den Bos, R., Jolles, J. W., \& Homberg, J. R. (2013). Social modulation of decisionmaking: a cross-species review. Frontiers in Human Neuroscience, 7, 1-16.

Boulinier, T., McCoy, K. D., Yoccoz, N. G., Gasparini, J., \& Tveraa, T. (2008). Public information affects breeding dispersal in a colonial bird: kittiwakes cue on neighbours. Biology Letters, 4, 538-540.

Boyd, R., \& Richerson, P. (1985). Culture and the evolutionary process. Chicago, IL: University of Chicago Press. 
Brown, C. R., Bomberger Brown, M., \& Danchin, E. (2000). Breeding habitat selection in cliff swallows: the effect of conspecific reproductive success on colony choice. Journal of Animal Ecology, 69, 133-142.

Chapman, B. B., Ward, A. J. W., \& Krause, J. (2008). Schooling and learning: early social environment predicts social learning ability in the guppy, Poecilia reticulata. Animal Behaviour, 76, 923-929.

Chou, L. S., \& Richerson, P. J. (1992). Multiple models in social transmission of food selection by Norway rats, Rattus norvegicus. Animal Behaviour, 44, 337-343.

Claidière, N., \& Whiten, A. (2012). Integrating the study of conformity and culture in humans and nonhuman animals. Psychological Bulletin, 138, 126-145.

Cohen, R., Bornstein, R., \& Sherman, R. C. (1973). Conformity behavior of children as a function of group makeup and task ambiguity. Developmental Psychology, 9, $124-131$.

Coolen, I., van Bergen, Y., Day, R. L., \& Laland, K. (2003). Species difference in adaptive use of public information in sticklebacks. Proceedings of the Royal Society B: Biological Sciences, 270, 2413-2419.

Corriveau, K. H., \& Harris, P. L. (2010). Preschoolers (sometimes) defer to the majority in making simple perceptual judgments. Developmental Psychology, 46, $437-445$.

Danchin, E., Boulinier, T., \& Massot, M. (1998). Conspecific reproductive success and breeding habitat selection: implications for the study of coloniality. Ecology, 79, 2415-2428.

Day, R. L., MacDonald, T., Brown, C., Laland, K. N., \& Reader, S. M. (2001). Interactions between shoal size and conformity in guppy social foraging. Animal Behaviour, 62, 917-925.

Deutsch, M., \& Gerard, H. B. (1955). A study of normative and informational social influences upon individual judgment. Journal of Abnormal and Social Psychology, 51, 629-636.

Dindo, M., Thierry, B., \& Whiten, A. (2008). Social diffusion of novel foraging methods in brown capuchin monkeys (Cebus apella). Proceedings of the Royal Society B: Biological Sciences, 275, 187-193.

Dindo, M., Whiten, A., \& de Waal, F. B. M. (2009). In-group conformity sustains different foraging traditions in capuchin monkeys (Cebus apella). PLoS One, 4 e7858.

Espmark, Y. O., Lampe, H. M., \& Bjerke, T. K. (1989). Song conformity and continuity in song dialects of redwings Turdus iliacus and some ecological correlates. Ornis Scandinavica, 20, 1-12.

Galef, B. G. (2009). Strategies for social learning: testing predictions from formal theory. Advances in the Study of Behavior, 39, 117-151.

Galef, B. G., \& Whiskin, E. E. (1995). Learning socially to eat more of one food than of another. Journal of Comparative Psychology, 109, 99-101.

Galef, B. G., \& Whiskin, E. E. (2008). 'Conformity' in Norway rats? Animal Behaviour, 75, 2035-2039.

Grüter, C., Leadbeater, E., \& Ratnieks, F. L. W. (2010). Social learning: the importance of copying others. Current Biology, 20, 683-685.

Haun, D. B. M., van Leeuwen, E. J. C., \& Edelson, M. G. (2013). Majority influence in children and other animals. Developmental Cognitive Neuroscience, 3, 61-71.

Haun, D. B. M., Rekers, Y., \& Tomasello, M. (2012). Majority-biased transmission in chimpanzees and human children, but not orangutans. Current Biology, 22, 727-731.

Haun, D. B. M., Rekers, Y., \& Tomasello, M. (in press). Great apes stick with what they know; children conform to others. Psychological Science.

Henrich, J., \& Boyd, R. (1998). The evolution of conformist transmission and the emergence of between-group differences. Evolution and Human Behavior, 19, 215-241.

Hopper, L. M., Schapiro, S. J., Lambeth, S. P., \& Brosnan, S. F. (2011). Chimpanzees' socially maintained food preferences indicate both conservatism and conformity. Animal Behaviour, 81, 1195-1202.

Hrubesch, C., Preuschoft, S., \& van Schaik, C. (2009). Skill mastery inhibits adoption of observed alternative solutions among chimpanzees (Pan troglodytes). Animal Cognition, 12, 209-216.

Jenness, A. (1932). The role of discussion in changing opinion regarding a matter of fact. Journal of Abnormal Social Psychology, 27, 279-296.

Jolles, J. W., de Visser, L., \& van den Bos, R. (2011). Male Wistar rats show individual differences in an animal model of conformity. Animal Cognition, 14, 769-773.

Kendal, R. L., Coolen, I., van Bergen, Y., \& Laland, K. N. (2005). Trade-offs in the adaptive use of social and asocial learning. Advances in the Study of Behavior, 35, 333-379.

Kendal, R. L., Coolen, I., \& Laland, K. N. (2004). The role of conformity in foraging when personal and social information conflict. Behavioral Ecology, 15, 269-277.

Kendal, R. L., Coolen, I., \& Laland, K. N. (2009). Adaptive trade-offs in the use of social and personal information. In R. Dukas, \& J. M. Ratcliffe (Eds.), Cognitive ecology II (pp. 249-271). Chicago, IL: University of Chicago Press.

King, A. J., \& Cowlishaw, G. (2007). When to use social information: the advantage of large group size in individual decision making. Biology Letters, 3, 137-139.

Konopasky, R. J., \& Telegdy, G. A. (1977). Conformity in the rat: a leader's selection of door color versus a learned door-color discrimination. Perceptual and Motor Skills, 44, 31-37.

Lachlan, R. F., Crooks, L., \& Laland, K. (1998). Who follows whom? Shoaling preferences and social learning of foraging information in guppies. Animal Behaviour, 56, 181-190.
Laland, K. N. (2004). Social learning strategies. Learning \& Behavior, 32, 4-14.

Laland, K., \& Williams, K. (1997). Shoaling generates social learning of foraging information in guppies. Animal Behaviour, 53, 1161-1169.

Latane, B., \& Wolf, S. (1981). The social impact of majorities and minorities. Psychological Review, 88, 438-453.

Leadbeater, E., \& Chittka, L. (2005). A new mode of information transfer in foraging bumblebees? Current Biology, 15, 447-448.

Leadbeater, E., \& Chittka, L. (2007a). The dynamics of social learning in an insect model, the bumblebee (Bombus terrestris). Behavioral Ecology and Sociobiology, 61, 1789-1796.

Leadbeater, E., \& Chittka, L. (2007b). Social learning in insects: from miniature brains to consensus building. Current Biology, 17, 703-713.

van Leeuwen, E. J. C., Call, J., \& Haun, D. B. M. (n.d.) [Human children rely more on social information than chimpanzees.] Unpublished raw data.

van Leeuwen, E. J. C., Cronin, K. A., Schütte, S., Call, J., \& Haun, D. B. M. (2013) Chimpanzees flexibly adjust their behaviour in order to maximize payoffs, not to conform to majorities. PLoS One, 8, e80945.

van Leeuwen, E. J. C., \& Haun, D. B. M. (2013). Conformity in primates: fad or fact? Evolution and Human Behavior, 34, 1-7.

MacLean, E. L., Matthews, L. J., Hare, B. A., Nunn, C. L., Anderson, R. C., Aureli, F., et al. (2012). How does cognition evolve? Phylogenetic comparative psychology. Animal Cognition, 15, 223-238.

Mery, F., Varela, S. A. M., Danchin, E., Blanchet, S., Parejo, D., Coolen, I., et al. (2009). Public versus personal information for mate copying in an invertebrate. Current Biology, 19, 730-734.

Mesoudi, A. (2009). How cultural evolutionary theory can inform social psychology and vice versa. Psychological Review, 116, 929-952.

Morgan, T. J. H., \& Laland, K. (2012). The biological bases of conformity. Frontiers in Neuroscience, 6, 87. http://dx.doi.org/10.3389/fnins.2012.00087.

Morgan, T. J. H., Rendell, L., Ehn, W., Hoppitt, W., \& Laland, K. (2012). The evolutionary basis of human social learning. Proceedings of the Royal Society B: Biological Sciences, 279, 553-662.

Mundinger, P. C. (1982). Microgeographic and macrogeographic variation in birds. In D. E. Kroodsma, E. H. Miller, \& H. Ouellet (Eds.), Acoustic communication in birds (pp. 147-208). New York, NY: Academic Press.

Payne, R. B. (1985). Behavioral continuity and change in local song populations of village indigobirds Vidua chalybeata. Journal of Comparative Ethology, 70, 1-44.

Perreault, C., Moya, C., \& Boyd, R. (2012). A Bayesian approach to the evolution of social learning. Evolution and Human Behavior, 33, 449-459. http://dx.doi.org/ 10.1016/j.evolhumbehav.2011.12.007.

Perry, S. (2009). Conformism in the food processing techniques of white-faced capuchin monkeys (Cebus capucinus). Animal Cognition, 12, 705-716.

Pesendorfer, M. B., Gunhold, T., Schiel, N., Souto, A., Huber, L., \& Range, F. (2009). The maintenance of traditions in marmosets: individual habit, not social conformity? A field experiment. PLoS One, 4, e4472.

Pike, T. W., \& Laland, K. N. (2010). Conformist learning in nine-spined sticklebacks foraging decisions. Biology Letters, 6, 466-468.

Price, E. E., Lambeth, S. P., Schapiro, S. J., \& Whiten, A. (2009). A potent effect of observational learning on chimpanzee tool construction. Proceedings of the Royal Society B: Biological Sciences, 276, 3377-3383.

Richerson, P. J., \& Boyd, R. (2005). Not by genes alone: How culture transformed human evolution. Chicago, IL: University of Chicago Press.

Rieucau, G., \& Giraldeau, L. A. (2011). Exploring the costs and benefits of social information use: an appraisal of current experimental evidence. Philosophical Transactions of the Royal Society B: Biological Sciences, 366, 949-957.

Rosa, P., Nguyen, V., \& Dubois, F. (2012). Individual differences in sampling behaviour predict social information use in zebra finches. Behavioral Ecology and Sociobiology, 66, 1259-1265.

van Schaik, C. P. (2012). Animal culture: chimpanzee conformity? Current Biology, 22, R402-R404.

Sherif, M. (1936). The psychology of social norms. Oxford, U.K.: Harper.

Starkweather, E. K. (1970). Starkweather social conformity test for preschool children. Stillwater, OK: Oklahoma State University.

Strimling, P., Enquist, M., \& Eriksson, K. (2009). Repeated learning makes cultural evolution unique. Proceedings of the National Academy of Sciences, 33, 13870-13874. http://dx.doi.org/10.1073/pnas.0903180106.

Tanford, S., \& Penrod, S. (1984). Social-influence model: a formal integration of research on majority and minority influence processes. Psychological Bulletin, 95, 189-225.

van de Waal, E., Borgeaud, C., \& Whiten, A. (2013). Potent social learning and conformity shape a wild primate's foraging decisions. Science, 340, 483-485.

Whiten, A. (1998). Imitation of the sequential structure of actions by chimpanzees (Pan troglodytes). Journal of Comparative Psychology, 112, 270-281.

Whiten, A., Horner, V., \& de Waal, F. B. M. (2005). Conformity to cultural norms of tool use in chimpanzees. Nature, 437, 737-740.

Wolf, M., Kurvers, R. H. J. M., Ward, A. J. W., Krause, S., \& Krause, J. (2013). Accurate decisions in an uncertain world: collective cognition increases true positives while decreasing false positives. Proceedings of the Royal Society B: Biological Sciences, 280. http://dx.doi.org/10.1098/rspb.2012.2777. 